\title{
Baja tolerancia a la frustración desde la terapia racional emotiva conductual
}

\author{
Low tolerance to frustration from rational emotional behavioral therapy \\ Baixa tolerância à frustração da terapia comportamental emocional racional
}

\author{
Abdel Yashin Avilés Sánchez \\ bdlvlssnchz@gmail.com \\ https://orcid.org/0000-0002-9960-9718 \\ Universidad Técnica de Babahoyo, Babahoyo-Ecuador
}

Recibido: junio 2021

Revisado: julio 2021

Aceptado: agosto 2021

Publicado: septiembre 2021

\section{RESUMEN}

Cuando una persona se enfrenta a una situación imprevista, la baja tolerancia a la frustración ocasiona que el individuo genere una serie de pensamientos que terminen desembocando en una sensación de incomodidad que a su vez provoque una serie de reacción que pueda acabar en hostilidad, por eso, este estudio de caso tuvo como objetivo general la identificación de las características de la baja tolerancia a la frustración desde el punto de vista de la terapia racional emotiva-conductual, se consideró factible, así que fue implementado como técnicas de recolección de información, la historia clínica, la observación y test psicométricos que apoyaron al diagnóstico del paciente y a desarrollar un esquema terapéutico el cual ayudará a mejorar la calidad de vida del paciente y demás personas que presenten este tipo de caso.

Palabras clave: Frustración; terapia racional; terapia emotiva; terapia conductual, hostilidad
When a person faces an unforeseen situation, the low tolerance for frustration causes the individual to generate a series of thoughts that end up leading to a feeling of discomfort that in turn provokes a series of reactions that can end in hostility, that is why, this case study had as a general objective the identification of the characteristics of low tolerance to frustration from the point of view of rational emotional-behavioral therapy, it was considered feasible, so it was implemented as information collection techniques, the clinical history, observation and psychometric tests that supported the diagnosis of the patient and to develop a therapeutic scheme which will help to improve the quality of life of the patient and other people who present this type of case.

Key words: Frustration; rational therapy; emotional therapy; behavioral therapy, hostility

\section{RESUMO}

Quando uma pessoa se depara com um imprevisto, a baixa tolerância à frustração faz com que o indivíduo gere uma série de pensamentos que acabam levando a um sentimento de desconforto que por sua vez provoca uma série de reações que podem culminar em hostilidade, por isso, este o estudo de caso teve como objetivo geral a identificação das características de baixa tolerância à frustração do ponto de vista da terapia emocional-comportamental racional, foi considerado viável, por isso foi implementado como técnicas de coleta de informações, a história clínica, observação e psicométrica testes que auxiliem no diagnóstico do paciente e no desenvolvimento de um esquema terapêutico que ajude a melhorar a qualidade de vida do paciente e de outras pessoas que apresentarem este tipo de caso.

Palavras chave: Frustração; terapia racional; terapia emocional; terapia comportamental, hostilidade 


\section{INTRODUCCIÓN}

Albert Ellis, otorgar el término "baja tolerancia a la frustración" o BTF como aporte a la terapia racional emotiva conductual (TREC). Lega (2017), conceptualiza la baja tolerancia como la imposibilidad que posee la persona con respecto a la postergación del placer o no tener capacidad de realizar sus actividades con calma. De este modo, se puede decir, que la baja tolerancia a la frustración, es la falta de capacidad que tiene el ser humano para superar inconvenientes que puedan interferir con la realización o culminación de actividades ya planeadas.

Las personas con baja tolerancia a la frustración por lo general buscan evitar las contrariedades que se puedan presentar, las cuales impida la realización de actividades, pero esto a su vez genera que la baja tolerancia a la frustración permanezca y se reafirme. En este sentido, Lega (2017), refiere que las personas con baja tolerancia se comportan de tal forma que pueden conseguir los objetivos que se plantean de forma inmediata, pero esto a su vez causa que en un futuro siga manteniendo estas creencias irracionales. Por ende, se puede entender que de una forma irónica el mantener este tipo de exigencias y evitaciones, lo que genera es acreditar esta cognición.

Sin embargo, cuando ocurre la disputa entre lo que se quiere lograr y eventos que no se esperaban, es cuando se produce un cumulo de emociones que luchan por hacer lo posible por seguir el plan trazado. Al respecto, Palacios (2013), dice que, "se enfrenta el deseo de un hecho y la imposibilidad de hacerlo este choque emocional es el que causa el estado de frustración" (p.228). Lo que se interpreta como una lucha interna de ideales a cumplir en ese momento y no dejar las cosas para después.

Es así, como nace la necesidad de desarrollar una investigación referente a la temática presente, por ende, el estudio pretende dar a conocer la terapia racional emotiva-conductual, estructura, modelo de intervención psicológica y como está es usada para la intervención, a través de un estudio de caso.

El estudio de caso, se lleva a cabo a un joven de 24 años de edad, de la ciudad de Vinces, trabajando en sus cogniciones las cuales mostraron un pensamiento irracional con respecto a la tolerancia a la frustración, planteándose el propósito de aumentar la tolerancia a la frustración, se logró recabar información sobre la baja tolerancia a la frustración y las características y la intervención bajo el punto de vista de la terapia racional emotiva conductual (TREC). De tal manera, el propósito de la investigación es, identificar la caracterización de la baja tolerancia a la frustración en un paciente del centro de especialidades "San Lorenzo "de Vinces.

El presente estudio otorga un aporte significativo a las personas con este tipo de problemas, ya que estarán siendo beneficiadas, además, este estudio de caso puede ser usado como una guía base para fututas investigaciones profesionales en el área psicológica y en relación al tema investigado, así como de forma directa está siendo beneficiado el paciente denominado como 
"Oliver" ya que por medio de la investigación e intervención psicológica se mejore la calidad de vida.

Cabe destacar, que se debe tomar en cuenta para el desarrollo de las investigaciones la relación entre la baja tolerancia a la frustración con la procrastinación, hostilidad, depresión y la baja tolerancia en niños.

La procrastinación para Angarita (2012), es el hecho de postergar o prolongar el cumplimiento de un compromiso o actividad pendiente. De forma que la baja tolerancia a la frustración puede manifestar el deseo de prolongar las actividades o tareas para no enfrentarse a la realidad de que puede sentirse incomodo de una forma exagerada al dudar de si poder lograrlas realizar con éxito.

A demás Angarita (2012) afirma que lejos de ser un problema que se circunscribe al ámbito académico, también se presentan en el ámbito muy diferentes como: el laboral, el cuidado en la atención de la familia o pareja enfermo, cuando una persona se está capacitando profesional e inclusive en el uso de su tiempo libre. Por tanto, se puede mencionar que la baja tolerancia a la frustración y la procrastinación interfieren en realizaciones con mucha relevancia y que de seguir aplazando las mismas, se tendría consecuencias graves.

Como en este caso la hostilidad, que la define Palmero (2008), "como una actitud negativa hacia los demás y hacia la misma naturaleza humana, que consiste en su enemistad, denigración y rencor" (p.30). De manera que, una vez presentada la baja tolerancia a la frustración, puede hacer que las personas reacciones manifestando palabras que denigren a personas a su alrededor.

Pero en cuanto reacciones se trata, si bien se ha mencionado que el sentirse con frustración ante un evento o estimulo conlleva una respuesta agresiva, esto no es una regla firme ya que puede desencadenar otros tipos de reacciones. "En humanos, al igual que en los otros animales, si bien los resultados tendieron a mostrar que la frustración genera agresión, las medidas dependientes utilizadas pueden dar lugar a interpretaciones ambiguas, en otros casos no desencadena respuestas agresivas" (Mustaca, 2018, p,71). Por ende, no siempre se tienen respuestas de esta índole en personas que no toleren la frustración.

Tomando en cuenta también lo mencionado anteriormente, se debe considerar la baja tolerancia a la frustración como propiciaste a sentimientos de tristeza. Mustaca, (2018), afirma que "la pérdida de un ser querido, del trabajo o problemas económicos pueden traer como consecuencia alteraciones en el área social, como mayor irritabilidad, depresión, agresividad" (p.66). Esto por crearse ideales que como ya se ha dicho con anterioridad al no cumplirse se obtienen las frustraciones.

Por último, en niños la baja tolerancia a la frustración con regularidad se presenta en forma de agresión, ya sea a los pares iguales, maestros y padres. Martínez (2008), mencionan que los impulsos de la agresión constatan que la forma agresiva de actuar es propiciada por el acto de paralización de las actividades y esto se debe a las frustraciones. Por consiguiente, no es relevante para el menor la persona que sea, si se siente con frustración, va a actuar de forma agresiva, en la mayoría de los casos. 


\section{METODOLOGÍA}

La presente investigación fue desarrollada bajo la modalidad estudio de caso, considerándose de esta manera, un estudio factible, ya que el paciente mostró una gran predisposición para colaborar en el caso. Utilizándose la entrevista estructurada para lo cual se utilizó la historia clínica como instrumento en la recolección de información, de igual forma se realizó la observación clínica para lo cual el instrumento tomado en cuenta fue la guía de observación, los test psicométricos y proyectivos, así como el inventario de depresión de Beck test de la persona bajo la lluvia, también el test de frustración de Rosenzweig.

Para la entrevista semiestructurada se utilizó el instrumento historia clínica, que para la psicología es uno de las principales herramientas, permitiendo así reunir todos los datos posibles del paciente de manera cronológica y tomando en cuenta los aspectos únicos de cada paciente y sus síntomas para brindar así un diagnóstico de manera más efectiva y por ende un mejor seguimiento. En la observación directa el método que se aplicó fue una guía de observación de esta forma se pudo analizar y describir los comportamientos que realizó el investigador y el observador pudo explicar y seguir de manera más específica un comportamiento además de registrarlo y usarlo como evidencia visual.

Adicional, se utilizaron test psicométricos entre ellos, el inventario de depresión de Beck, el objetivo es medir los tipos e intensidad de la depresión en una persona tomando en cuenta las últimas 2 semanas, y los test proyectivo, lo cual, son evaluaciones que se encargan de medir aspectos de la personalidad del individuo, así como emociones y conflictos internos, se representan en general por medio de dibujos u otras expresiones artísticas, entre ellos, se aplicaron el test de la persona bajo la lluvia, con el fin de resaltar las cualidades de una persona y su reacción ante una circunstancia estresante como es la lluvia, de esta manera se pudo ver evidencia de los rasgos que posee la persona para afrontar dichos eventos. $Y$ el test de frustración de Rosenzweig, se utilizó para medir las respuestas del individuo ante circunstancias frustrantes, está constituido por 24 imágenes donde sitúa al paciente en distintos eventos donde la frustración podría jugar un papel protagónico.

\section{DESCRIPCIÓN DEL CASO CLÍNICO}

El presente estudio de caso se llevó a cabo a un joven de 24 años de edad, soltero, en la actualidad cursando una carrera universitaria, oriundo del cantón Vinces, provincia de los Ríos de Ecuador y que asiste a consulta por iniciativa propia, para protección de identidad y con el permiso correspondiente del paciente, se lo denominará como "Oliver", se realizó un número de 6 sesiones que pasaran a ser detalladas a continuación (Tabla 1 ). 
Tabla 1. Resultados de las sesiones.

\begin{tabular}{|c|c|c|c|c|}
\hline Sesión & Fecha & Tiempo & Instrumento aplicado & Información obtenida \\
\hline 1 & 03 Julio & 45 minutos & Historia clínica & $\begin{array}{l}\text { Oliver expresó "cuando no me sales las cosas } \\
\text { como quiero siento mucho odio y me desquito } \\
\text { con cualquier persona que pase" }\end{array}$ \\
\hline \multirow[t]{2}{*}{2} & 16 Julio & 45 minutos & $\begin{array}{l}\text { - Terapia Racional Emotiva- } \\
\text { Conductual. }\end{array}$ & Se estableció un diagnóstico para el paciente \\
\hline & & & - Modelo A, B, C & \\
\hline \multirow[t]{3}{*}{3} & 22 Julio & 45 minutos & $\begin{array}{l}\text { - Test de la persona bajo la } \\
\text { lluvia. }\end{array}$ & Se estableció un diagnóstico para el paciente \\
\hline & & & $\begin{array}{l}\text { - Inventario de depresión } \\
\text { de Beck. }\end{array}$ & \\
\hline & & & $\begin{array}{l}\text { - Test de frustración de } \\
\text { Rosenzweig }\end{array}$ & \\
\hline 4 & & 45 minutos & $\begin{array}{l}\text { Retroalimentación de las } \\
\text { sesiones pasadas }\end{array}$ & $\begin{array}{l}\text { Se procedió a crear un inventario basándose en } \\
\text { las interpretaciones del paciente con respecto a } \\
\text { las cosas terribles contra las cosas normales que } \\
\text { pueden pasar en la semana para luego realizar } \\
\text { un ejercicio de evaluación con puntaje del uno } \\
\text { al cien de cosas malas y compararla con cosas } \\
\text { realmente malas. }\end{array}$ \\
\hline 5 & 10 Agosto & 45 minutos & $\begin{array}{l}\text { Retroalimentación de las } \\
\text { sesiones pasadas }\end{array}$ & $\begin{array}{l}\text { Se le explico a Oliver que se iba a trabajar en una } \\
\text { técnica para regular las emociones, se pidió al } \\
\text { paciente que mediante la imaginación se coloque } \\
\text { en una situación que le causara frustración, y } \\
\text { cuando sienta emociones fuertes, las cambiara } \\
\text { por unas que sean más fácil de sobrellevar. }\end{array}$ \\
\hline 6 & 19 Agosto & 45 minutos & $\begin{array}{l}\text { Retroalimentación de las } \\
\text { sesiones pasadas }\end{array}$ & $\begin{array}{l}\text { Se encomendó labor para casa, sobre buscar y } \\
\text { quedarse en situaciones estresantes y aumentar } \\
\text { de forma gradual el tiempo de exposición para } \\
\text { así aumentar la tolerancia. }\end{array}$ \\
\hline
\end{tabular}

Adicional, se muestra en la tabla 2 , los resultados obtenidos posterior a la aplicación de los diversos test proyectivos.

En el test de la persona bajo la lluvia, el paciente elaboró un dibujo con trazos, secuencias, dimensiones y otros aspectos característicos de personas con una agresividad leve, exigentes en sí misma, y con muy baja tolerancia a las frustraciones. Por otro lado, el resultado obtenido por el test de frustración de Rosenzweig, correspondieron a una persona con hostilidad, reproche hacia otras personas y hacia sí mismo, y nuevamente se evidenció la baja tolerancia a las frustraciones. Mientras que en el último test (inventario de depresión de Beck), no se evidenció cuadro depresivo. 
Tabla 2. Resultados de las sesiones.

\begin{tabular}{|c|c|c|}
\hline Tipo de Test & Test & Situación detectada \\
\hline \multirow[t]{5}{*}{ Proyectivos } & Test de la persona bajo la lluvia & - Leves impulsos de agresividad. \\
\hline & & $\begin{array}{l}\text { - No tiene manejo con respecto a la resolución de } \\
\text { conflicto. }\end{array}$ \\
\hline & & $\begin{array}{l}\text { - Muestra una auto insatisfacción causada por } \\
\text { exigencias hacia sí mismo. Baja tolerancia a las } \\
\text { frustraciones. }\end{array}$ \\
\hline & & - Susceptible ante las situaciones estresantes. \\
\hline & & - Auto exigente. \\
\hline \multirow[t]{4}{*}{ Proyectivos } & Test de frustración de Rosenzweig: & - Persona con hostilidad \\
\hline & & - Reproche hacia sí mismo y otras personas. \\
\hline & & - Espera que sus problemas sean resueltos por otros. \\
\hline & & - Baja tolerancia a la frustración. \\
\hline \multirow[t]{4}{*}{ Proyectivos } & Inventario de depresión de Beck & $\begin{array}{l}\text { - Área somática: Aumento de ritmo cardíaco y } \\
\text { sudoración }\end{array}$ \\
\hline & & $\begin{array}{l}\text { - Área afectiva: Tristeza y desanimo al no lograr lo } \\
\text { propuesto. }\end{array}$ \\
\hline & & $\begin{array}{l}\text { - Área cognitiva: El paciente tiene la idea de no ser } \\
\text { capaz de controlar su ira, pensamiento irracional }\end{array}$ \\
\hline & & $\begin{array}{l}\text { - Área conductual: Evita episodios frustrantes y de } \\
\text { esta manera se aísla en ocasiones. }\end{array}$ \\
\hline
\end{tabular}

Una vez, presentado los resultados obtenidos por medio de los instrumentos aplicados fue fundamental ejecutar un plan psicoterapéutico de acuerdo a los lineamientos de la terapia racional emotiva-conductual, con el fin de proporcionarle al paciente un mejor estilo de vida y modificar las estructuras de pensamientos que le generan malestar. El procedimiento a seguir trabajó en las tres áreas que fueron, el área cognitiva, el área conductual y el área emocional. Los mismo fueron presentados en la Tabla 3. 
Tabla 3. Lineamientos de la terapia racional emotiva-conductual.

\begin{tabular}{|c|c|c|c|c|c|}
\hline HALLAZGOS & ÁREA & META PSICOTERAPEUTICA & TÈCNICAS & $\begin{array}{c}\mathbf{N}^{\circ} \\
\text { SESIONES }\end{array}$ & RESULTADOS ESPERADOS \\
\hline $\begin{array}{l}\text { El paciente } \\
\text { mantiene la } \\
\text { idea de no } \\
\text { ser capaz de } \\
\text { controlar su ira }\end{array}$ & $\begin{array}{c}\text { Área } \\
\text { cognitiva }\end{array}$ & $\begin{array}{l}\text { Se enfocó en identificar y logra una } \\
\text { reflexión (Insight intelectual) del } \\
\text { paciente con respecto a las creencias } \\
\text { irracionales. }\end{array}$ & $\begin{array}{l}\text { Crear de acuerdo al pensamiento del } \\
\text { paciente una escala de cosas terribles que } \\
\text { pudieran pasar en torno a una semana. } \\
\text { Objetivo identificar las situaciones que el } \\
\text { paciente cree que son incapaces de tolerar. }\end{array}$ & 1 & $\begin{array}{l}\text { Se pudo utilizar de forma efectiva las } \\
\text { mismas creencias irracionales del paciente } \\
\text { obtenido como resultado la reflexión y } \\
\text { aceptación que existen escenarios más } \\
\text { desalentadores. }\end{array}$ \\
\hline
\end{tabular}

Crear una comparación entre las cosas terribles con anterioridad y ponerlas a prueba contra cosas realmente malas, Se realizó con el objetivo de que reflexione (insight intelectual).

El paciente comentó que se ha llegado a sentir con

tristeza,

desanimo e ira

El paciente cuando piensa que puede presentarse

un episodio

frustrante,

lo evita y de

esta manera

en ocasiones

se termina

aislando
Área Obtener una reflexión con respecto a

emocional las emociones que puedan causar un evento frustrante.

Analizar alternativas más racionales de cómo reaccionar ante eventos que el paciente cree difíciles de superar, para así aumentar la tolerancia a la frustración.
Imaginación racional-emotiva, ya que tiene por objetivo exponer de forma segura $y$ controlada al paciente mediante el uso de la imaginación a situaciones que evocan sentimientos intensos y a partir de esto pedir al paciente que intente cambiar esos sentimientos por unos más racionales.

Ensayo de conducta desalentadores.

1 El paciente aminoró la carga emocional experimentada, ya que se dio cuenta que una vez pensando que los eventos son difíciles, pero no imposibles, dio paso a que reflexionara de manera emocional.

El paciente pudo crear conductas racionales de afrontamiento ante eventos frustrantes gracias al ensayo de conducta y se busca el reforzamiento de esta conducta mediante la terea enviada a casa. 


\section{CONCLUSIONES}

En el presente estudio de caso se concluye que la baja tolerancia a la frustración puede presentarse desde la niñez y en la adultez puede ser el desencadenante de respuestas agresivas. A demás, se pudo identificar en el paciente reacciones de baja tolerancia a la frustración y hostilidad, que ya previamente se había tratado en la parte investigativa, de la misma manera se ubicó procrastinación en el paciente, puesto que aplazaba sus actividades y se quedaba en su cuarto sin salir, se demostró de esta manera que, aunque si hay relación de la baja tolerancia a la frustración con la depresión, pues el paciente no presentó dicho trastorno, esto se verificó con el inventario de depresión de Beck, el cual no cumplió con el criterio de diagnóstico.

Se corroboro también la existencia de creencias irracionales del paciente durante el trascurso de la sesión ya que el paciente creía que era imposible lograr calmarse durante un evento frustrante y pensaba que la sensación de frustración en sí era exageradamente grande, para identificar estas creencias fue necesario el modelo $A B C$ de la terapia racional emotiva-conductual, ya que su estructura si bien se encarga de encontrar un evento activador que genera una emoción para crear una consecuencia, la filosofía de la misma se sustenta en que son los pensamiento e interpretaciones de las personas lo que genera una consecuencia.

Tomando en consideración lo antes mencionado, hay que destacar las técnicas para modificar las conductas, emociones y pensamientos, ya que para este caso formaron parte fundamental para lograr estabilizar al paciente.

Para finalizar, se recomienda que el paciente continúe efectuando el ejercicio enviado a casa para incrementar la tolerancia a la frustración, aumentando el tiempo de exposición de forma gradual, se recomienda también la concurrencia de practicantes en el Centro de Especialidades "San Lorenzo" de Vinces, para proporcionar ayuda a esa comunidad y mejorar así el estilo de vida de los habitantes.

\section{REFERENCIAS}

Angarita, L. (2012). Aproximación a un concepto actualizado de la procrastinación. Revista Iberoameicana de psicologia, 5(2), 8594. Recuperado de https://revistas.ibero. edu.co/index.php/ripsicologia/article/ download/249/217

Lega, L. (2017). Terapia Racional Emotiva Conductual: Una versión teóricopráctica actualizada. Barcelona,España.: Paidós:psicologia, psiquiatria, Psicoterapia

Martínez, J. (2008). El comportamiento agresivo y algunas características a modificar en los niños y niñas. Investigaciones Andina, 10(16), 92-105. Recuperado de https:// $\mathrm{n} 9 . \mathrm{cl} / 56490$

Mustaca, A. (2018). Frustración y conductas sociales. Avances en Psicología Latinoamericana, 31(1), 61-85. Recuperado de https://dialnet.unirioja.es/ descarga/ articulo/6231480.pdf

Palacios, F. (2013). Depresión siglo XXI. Palibrio. Recuperado de https://n9.cl/ruqcq

Palmero, J. (2008). Hostilidad, psicofisiología y salud cardiovascular. Suma Psicologia., 14(1), 23-50. Recuperado de https://n9.cl ge6d8 